\title{
Anchor-Free Localization Using a Deep Neural Network in Wireless Sensor Networks with Multiple Sinks
}

This paper was downloaded from TechRxiv (https://www.techrxiv.org).

\section{LICENSE}

CC BY-SA 4.0

SUBMISSION DATE / POSTED DATE

04-08-2021 / 09-08-2021

\section{CITATION}

Mahapatro, Arunanshu; Rayavarapu, V CH Sekhar Rao (2021): Anchor-Free Localization Using a Deep Neural Network in Wireless Sensor Networks with Multiple Sinks. TechRxiv. Preprint. https://doi.org/10.36227/techrxiv.15104223.v1

$\mathrm{DOI}$ 


\title{
Anchor-Free Localization Using a Deep Neural Network in Wireless Sensor Networks with Multiple Sinks
}

\author{
V CH Sekhar Rao Rayavarapu, Member, IEEE, Arunanshu Mahapatro, Senior Member, IEEE and Amiya Kumar \\ Tripathy, Member, IEEE
}

\begin{abstract}
Wireless sensor networks (WSNs) is one of the vital part of the Internet of Things (IoT) that allow to acquire and provide information from interconnected sensors. Localization-based services are among the most appealing applications associated to the IoT. The deployment of WSNs in the indoor environments and urban areas creates obstacles that lead to the Non-Line-of-Sight (NLOS) propagation. Additionally, the localization accuracy is minimized by the NLOS propagation. The main intention of this paper is to develop an anchor-free node localization approach in multi-sink WSN under NLOS conditions using three key phases such as LOS/NLOS channel classification, range estimation, and anchor-free node localization. The first phase adopts Heuristicbased Deep Neural Network (H-DNN) for LOS/NLOS channel classification. Further, the same H-DNN is used for the range estimation. The hidden neurons of DNN are optimized using the proposed Adaptive Separating Operator-based Elephant Herding Optimization (ASO-EHO) algorithm. The node localization is formulated as a multi-objective optimization problem. The objectives such as localization error, hardware cost, and energy overhead are taken into consideration. ASO-EHO is used for node localization. The suitability of the proposed anchor-free node localization model is validated by comparing over the existing models with diverse counts of nodes.
\end{abstract}

Index Terms-Localization, IoT, ASO-EHO, H-DNN, NLOS/LOS

\section{INTRODUCTION}

The Internet of Things (IoT) paradigm generally focuses on presenting hundreds of small, interconnected units that can collaborate for a common goal. The growing number of these small connected devices allows IoT to become a reality. They are intelligent yet simple objects with sensing and wireless communication capabilities. WSN as an integral part of the IoT has been recognized as a promising data acquisition tool. The location of sensor nodes within the network plays a vital role regardless of the application and deployment. The process of determining the position of each sensor node is called localization. Localization can be accomplished through many techniques based on communication between anchor nodes with known location and the sensor node whose location has to be determined. However, there may be obstacles within indoor environments and urban areas that can block the LOS

V CH Sekhar Rao Rayavarapu is with Department of Electeronics and Telecommunication Engineering, VSSUT, Burla

Arunanshu Mahapatro is with Department of Electeronics and Telecommunication Engineering, VSSUT, Burla

Amiya Kumar Tripathy is with Department of Computer Science and Engineering, Don Bosco Institute of Technology Kurla-West, Mumbai connection between the nodes and leads to NLOS propagation. Localization performance is affected by the distance calculation errors introduced by NLOS propagation [1].

In WSN, the localization techniques are classified as rangefree localization techniques and range-oriented localization techniques. In the case of range-oriented localization techniques, several measurement approaches for the localization are categorized as angle of arrival (AOA) [2], time of arrival (TOA) [3], and received signal strength (RSS) [4]. The rangefree localization techniques do not calculate the angle or distance among the nodes. The positions are estimated by these techniques on the basis of measurement history distribution and network connectivity. The NLOS paths create challenges for range estimation-oriented techniques and need to be mitigated. NLOS propagation introduces positive biases in distance estimation algorithms, affecting localization accuracy significantly [5]. It is therefore important to identify and classify NLOS and LOS signals and to consider the influence of NLOS conditions on localization processes and develop strategies to minimize their impacts.

Classification issues are becoming increasingly suited to Machine Learning (ML) technologies. Using ML-based techniques to detect LOS/NLOS conditions is more likely to generate more accurate results since ML methods can extract patterns from many dimensions of channel data [5]. Non-parametric ML approaches such as Relevance Vector Machine (RVM) and Support Vector Machine (SVM) are more robust and precise for LOS/NLOS detection, where the received power, maximum power, rise time, kurtosis, and delay spread are extracted as training features [6], [7]. SVM suffers from shortcomings like high computational cost and non-probabilistic output when the training data count is huge. In comparison to the SVM, the RVM generally takes more training time but may be evaluated faster in practice. In recent years, Neural Networks (NNs) training has been improved, in which fully dimensional data, such as waveforms, images, and so on are given into a classification algorithm without any input transformations or complex feature extractions [8]. Deep learning is capable of addressing multidimensional optimization problems and allowing not just adaptive self-learning of complex, non-linear relations or features in a given dataset, but also a prediction of their values [9]. The Convolutional Neural Networks (CNNs) [10], along with their subset is possible for the production-ready usage owing to the enhancements in the deep learning $\mathrm{NN}$ training mechanisms. 
The nodes closest to the sink node forward the complete network traffic to the sink in single sink multi-hop wireless sensor networks. As a result, they deplete their energy faster, and energy-holes form near the sink node, reducing the network's lifetime. Multiple sink node deployment strategies for better load balancing are presented in literature to increase network lifetime and reduce packet delay. The use of multi-sink WSNs is becoming more common as a result of the significant advantages in network lifetime and energy balance[33], [34].

The anchor nodes represent the sensor nodes, in which the location coordinates, are positioned by the GPS or in a manual manner. The GPS-oriented anchor node configuration is inefficient in indoor environments, in which RF signals cannot reach from the satellites. The research of localization schemes without anchor nodes, known as anchor-free localizations, is important for these applications as well as for assisting the anchor-oriented localization scheme.

The major contributions of the paper are as follows:

- The novel H-DNN is used for LOS/NLOS classification, in which the hidden layers of the DNN are optimized to maximize accuracy and precision.

- The H-DNN is utilized for range estimation, and the ranging error estimates are used in the localization processes to remove the predicted error from the estimated ranges.

- A novel optimization approach known as ASO-EHO is developed for node localization.

- Anchor-free node localization is formulated as a multiobjective optimization problem with specific objectives such as localization error, hardware cost, and energy overhead.

The organization of the paper is as follows: The literature survey is described in Section II. Section III explains the system model. Anchor-free localization in WSN under the NLOS conditions is presented in Section IV. Proposed architectural model for anchor-free node localization with NLOS is presented in Section V. Section VI presents node localization using ASO-EHO. Results and discussions are presented in Section VII. Finally, Section VIII concludes the paper.

\section{Literature SURVEY}

\section{A. Related Works}

Bregar et al. [11] established two approaches for reducing localization error in indoor NLOS conditions using raw channel impulse response (CIR) information obtained from ultrawide band radios, which need no prior knowledge of the radio environment. These techniques were dependent on the ranging error regression and NLOS channel classification models. CNNs were used in both of these approaches. Koledoye et al. [12] proposed a method for evaluating measurement noise and NLOS biases based on fixed node placement. Hua et al. [13]] have introduced a new NLOS mitigation approach, where the geometric relationship among a mobile and base station was utilized. The range measurements that were damaged by the NLOS errors were identified and the position was estimated by the mobile station via the LOS measurements. Further, the NLOS detector threshold was derived. The localization was attained by a two-step weighted-least-squares algorithm, where both the angle and range measurements were taken into account. Jee et al. [14] have developed a new technique for the indoor wireless sensor node localization by means of visible light in NLOS condition. The NLOS condition was identified by the developed technique in a sensor environment, and the sensor nodes were localized in a subsequent manner. The visible light was employed for the localization process. Initially, the reflection points were localized by means of the time difference on arrival (TDOA). The sensor node location was described by means of the reflection and range angles that were calculated with the help of the new geometric techniques.

Yang et al. [15] proposed a NLOS identification technique with an import vector machine (IVM)-based localization algorithm and feature selection scheme. The feature selection scheme has improved the classification accuracy. When the probability output of the IVM was used with the localization algorithm, better positioning accuracy was found when compared to other approaches such as RVM and SVM. Musa et al. [16] have developed an NLOS detection technique by means of recursive decision tree learning. The approach was proposed using information from the UWB channel quality indicators as well as the modified priors splitting criterion and the Gini index. Wang et al. [17] have addressed a NLOS localization technique on the basis of residual analysis for minimizing the NLOS error. The distance was estimated using the TOA measurement model. The NLOS measurement was recognized via the residual analysis technique. The localization objective function was established by the LOS measurements. The particle swarm optimization with a constriction factor (PSOC) was developed for calculating the unknown node position. Wang et al. [18] have labelled the time-of-arrivalbased localization problem in the path of mixed LOS/NLOS conditions. On the basis of known path status, a novel RWLS technique was developed for enhancing the performance of the traditional robust weighted least squares (RWLS) technique.

\section{B. Review}

CNN [11] guarantees greater scalability and achieves less latency in the localization. But, the ranging error regression models and NLOS classification are not constructed, and extra extensive measurement campaigns are not produced in distinct indoor environments for capturing the NLOS and LOS propagation conditions. MDS scheme [12] permits the larger node deployments, and also symmetry is exhibited that permits the re-computing of precise anchor positions. Geometry-based NLOS [13] is applicable for the real-world scenarios and also describes the detection threshold by exploiting a semianalytical method. Yet, heavy calibration is required. VLC [14] provides less implementation cost and less hardware complexity, and expensive hardware is not needed. But, the localization error is not minimized by considering multiple reflections and employing the conditioned geometric methods. Import vector machine [15] contains least sparsity with less import vectors and is an effective and robust technique. But, time synchronization is required. DT [16] employs the outcomes for the UWB indoor location tracking accuracy enhancement and is employed for the ranging result elimination and bias correction. Yet, various severity conditions, 


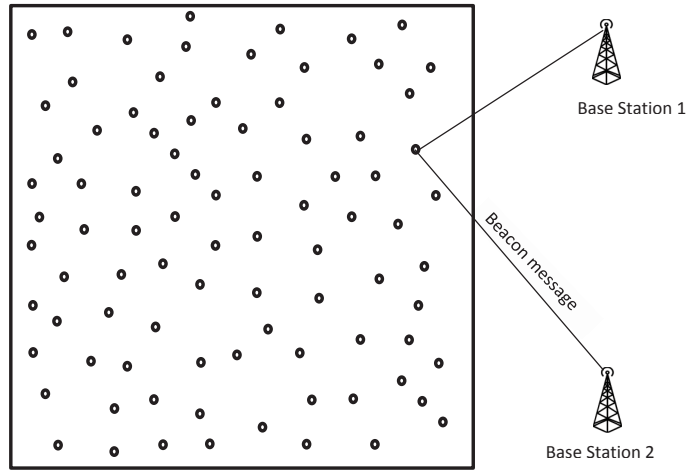

(a)

Fig. 1. Network Model.

the multi states NLOS is not detected. PSO [17] estimates the distance by the TOA and also minimizes the influence of the NLOS. But, the residual analysis method is not altered to enhance the efficiency of the particle filter. Robust weighted least squares (RWLS) [18] enhances the performance under the mixed NLOS/LOS conditions. Still, it needs more time synchronization. Hence, these challenges have motivated to develop an indoor localization in WSNs using novel machine learning and heuristic methods.

\section{SYSTEM MODEL}

\section{A. Network Model}

The proposed algorithm considers a network with $n$ sensor nodes non-uniformly distributed in a square area of side $L$, which is much larger than the communication range $\left(r_{t x}\right)$ of the sensor nodes. Every node maintains a neighbor table $N(\cdot)$. As illustrated in Fig. 1, two sink nodes are considered. Beacon messages are broadcast by sink nodes to all nodes in the network. As seen in Fig. 1(b), the nodes having LOS paths to both sink nodes are identified.

\section{B. Energy Consumption Model}

This paper, like [20], uses a basic model for radio hardware energy dissipation. The energy dissipated by the transmitter is used to power the radio electronics and the power amplifier. The energy dissipated by the receiver is used to power the radio equipment. Both the free space ( $D^{2}$ power loss) and the multi-path fading ( $D^{4}$ power loss) channel models are used, depending on the distance between the transmitter and receiver. The energy spent for transmission of an $r$-bit packets over distance $D$ is:

$E_{T x}(r, D)=r E_{\text {elec }}+r \epsilon D^{\alpha}= \begin{cases}r E_{\text {elec }}+r \epsilon_{f s} D^{2} & D<D_{0} \\ r E_{\text {elec }}+r \epsilon_{\text {amp }} D^{4} & D \geq D_{0}\end{cases}$

The electronics energy, $E_{\text {elec }}$, depends on factors such as the digital coding, and modulation. The amplifier energy, $\epsilon_{f s} D^{2}$

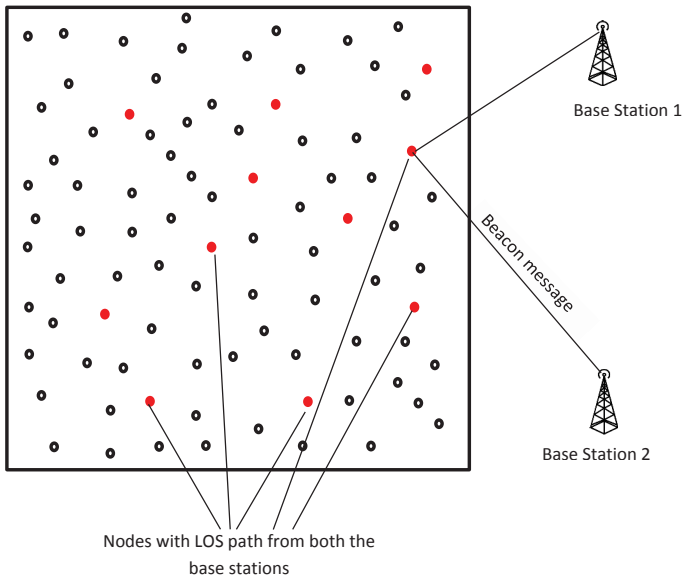

(b)

or $\epsilon_{a m p} D^{4}$, depends on the transmission distance and the acceptable bit-error rate. To receive this message, the radio expends energy:

$$
E_{R x}(r)=r E_{\text {elec }}
$$

\section{NLOS-BASED LOCALIZATION PROBLEM IN WSNS}

The localization issue is among the most researched difficulties in WSN because if the position of sensor nodes is unknown, coverage, power, and routing would be poor [19]. Because of the existence of NLOS in the real-time environment, the accuracy of WSN localization is reduced. In the measuring procedure, there are two types of localization errors. The first is measurement-related system error, such as accuracy, quantization error, and thermal noise. The second type of error is the NLOS error, which is caused by barriers between the base and the target station, causing signal spread to be manipulated. Hence, the two key issues in the localization are the NLOS error elimination and the basic WSN localization algorithm.

The suggested localization methods for WSN in literature, with anchor and unknown nodes, are carried out in a twophases. The first phase is known as the ranging phase, and it is where algorithms calculate the distance between unknown nodes and neighbouring anchor nodes. In the second phase, the location of the nodes is calculated using various methodologies and the range information obtained from previous phase. However, because the deployment of anchor nodes in some WSN applications may be impractical, thus, anchor-free node localization is a viable solution.

\section{A. Problem Formulation}

The goal of the WSN localization issue with $n$ sensor nodes is to estimate the positions of $n$ unknown nodes using location information from two or more sink nodes. Each sink node has a transmission range $R$ such that every senosr node in the network can hear the sink nodes' beacon messages. A sensor node is deemed to be localized if it is within transmission 
range of two or more sink nodes. This is a $2 D$ localization issue with $2 n$ unknown coordinates.

In this article, two sink nodes are considered, and the RSS technique is used to calculate the distance between nodes as well as the distance between nodes and the sink node. Imprecise measurements can occur regardless of the ranging method used. The NLOS as well as the measurement error seems to be independent, and hence it can be separated from the distance measurement. Each node estimates the distance $d_{i}$ from $i^{t h}$ sink node where $i=1,2$.

$$
d_{i_{N L O S}}=d_{i}+e_{i}+e_{N L O S}
$$

where $d_{i}=\sqrt{\left(x-x_{i}\right)^{2}+\left(y-y_{i}\right)^{2}}$ is the actual distance calculated, $n_{i}$ is the Gaussian noise, and $e_{N L O S}$ is the NLOS error. The NLOS error is a positive random variable.

To counter the affect of $e_{N L O S}$, LOS/NLOS classification is vital. The estimation of the coordinates of the $N$ unknown nodes can be expressed as a multiobjective optimization problem involving the minimization of objectives such as localization error, hardware cost, and energy overhead.

\section{B. Calculation of Objectives}

1) Localization error: The error is calculated by comparing the nodes' current and actual location. To improve localization, the error must be minimized. The average localization error is given by

$$
E r r=\frac{\sum_{i=1}^{n} \sqrt{\left(\hat{x}_{i}-x_{i}\right)^{2}+\left(\hat{y}_{i}-y_{i}\right)^{2}}}{n}
$$

Where, the desired coordinate of the unknown node is represented as $\left(x_{i}, y_{i}\right)$, the estimated coordinate of the unknown node is represented as $\left(\hat{x}_{i}, \hat{y}_{i}\right)$.

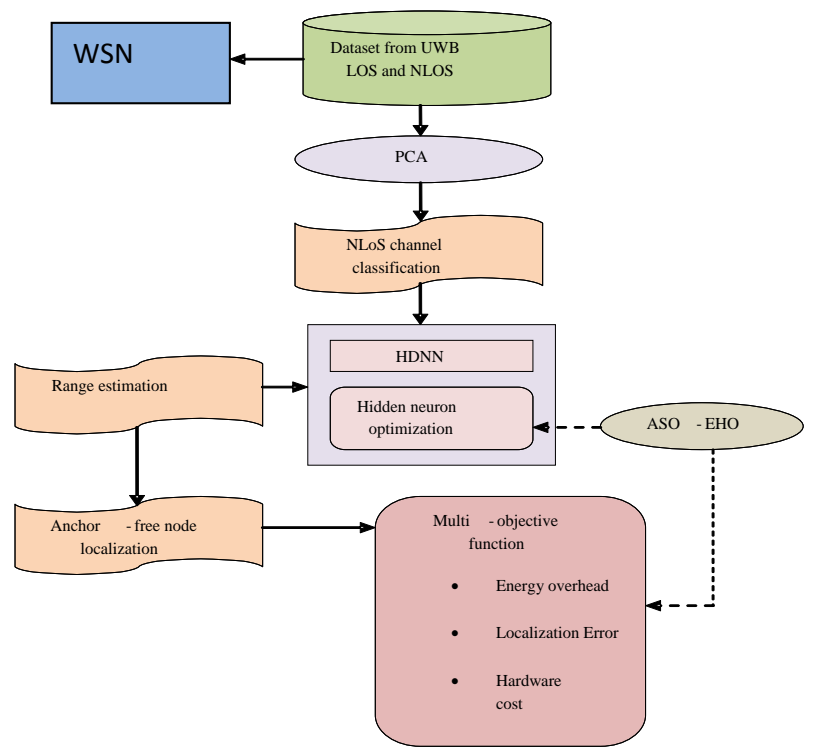

Fig. 2. Proposed Architecture
2) Energy Overhead: If more energy is spent during the localization process, the network lifetime of the WSN is reduced. In an n-node WSN, each node has a unique identifier which can be encoded with $\log _{2} n$ bits. As previously stated, each node in the network receives the beacon message from the two sink nodes, which is represented by $z$ number of bits. The energy dissipated in receiving the first beacon message is $n\left(2 z+\log _{2} n\right)\left(E_{R x}\right)$. After receiving the beacon message each node executes the classification algorithm. If the energy spent in the classification is represented gy $E_{\text {classi }}$ then the energy dissipated is $n E_{\text {classi }}$. The $r_{1}$ number of nodes with LOS path to both the sink nodes estimates its coordinates and spend energy of $\left(r_{1}\right) E_{\text {est }}$, where $E_{\text {esti }}$ is the energy spent in range estimation and node localization. These $r_{1}$ nodes broadcasts beacon message amounting an energy cost of $r_{1}\left(z+\log _{2} r_{1}\right)\left(E_{T x}\right)$. Each neighbours of these $r_{1}$ nodes will receive the beacon message by spending energy of $\sum_{i=1}^{r}|N(i)| E_{R x}$. Upon receiving the beacon messages $\sum_{i=1}^{r}|N(i)|$ nodes spend energy of $\sum_{i=1}^{r}|N(i)| E_{\text {classi }}$ for classification. Let $r_{2}$ number of nodes have LOS path to at least two of their neighbours. These $r_{2}$ number of nodes do the range estimation and localization by spending energy of $r_{2} E_{\text {est } i}$ and broadcast the beacon message by spending energy $r_{2}\left(z+\log _{2} r_{2}\right)\left(E_{T x}\right)$. This process continues till all the nodes in the WSN are localized. Therefore, the total energy overhead is given by

$$
E_{T}=n\left(2 z+\log _{2} n\right)\left(E_{R x}\right)+n E_{\text {classi }}+\sum_{j} E_{r_{j}}
$$

where $E_{r_{j}}=\sum_{i=1}^{r_{j}}\left(|N(i)| E_{R x}+|N(i)| E_{\text {classi }}\right)+r_{j+1} E_{\text {est } i}+$ $r_{j}\left(z+\log _{2} r_{j+1}\right)\left(E_{T x}\right)$.

3) Hardware cost : It is defined as, the total cost required for moving one node from the current location to another optimal location. It must be kept to a minimum in order to improve the efficacy of localization. Here, the hardware cost is evaluated by defining a specific cost for the distance between the nodes.

\section{PROPOSED ARCHITECTURAL MODEL FOR ANCHOR-FREE NODE LOCALIZATION WITH NLOS}

\section{A. Proposed Architecture}

The proposed approach considers three phases: NLOS channel classification, range estimation, and anchor-free node localization. Initially, H-DNN is trained by using the standard publicly accessible UWB LOS and NLOS datasets [21]. For the dimensionality reduction, the collected attributes are subjected to PCA. The LOS/NLOS channel is subsequently classified using the H-DNN algorithm. The suggested ASOEHO optimizes the hidden neurons of DNN with the goal of maximizing accuracy and precision. In the second phase, known as range estimation, the same H-DNN is employed. Range estimate is performed on nodes that have a LOS path to both sink nodes. The suggested ASO-EHO is used for node localization in the final phase. Once the coordinates of the nodes with a LOS path to both sink nodes have been estimated, these nodes will act as anchor nodes for other nodes that do not have a LOS path to both sink nodes and will broadcast a 
beacon message. Nodes with estimated coorinates are labelled as known, while others are labelled as unknown. When the unknown node receives the beacon message from the known node, it performes the three phases listed above. This process will be repeated until all nodes in the network have been designated as known nodes. The architecture of the proposed anchor-free node localization under the NLOS condition is shown in Fig. 2.

\section{B. Dataset Description}

The dataset used here for anchor free localization in WSN under the NLOS environment is the standard publically available UWB LOS and NLOS dataset [21]. It was created using the SNPN-UWB board and the DecaWave DWM1000 UWB radio module. The measurements were taken from seven different indoor locations, including a boiler room, a bedroom, a kitchen with a living area, a small workshop, a small apartment, office 2, and office 1. 3000 LOS samples and 3000 NLOS samples were collected from each indoor site. Distinct locations were chosen to avoid the development of locationspecific NLOS and LOS models. In all, 42000 samples were considered, including 21000 for the NLOS channel condition and 21000 for the LOS channel condition. The samples are taken in a random manner for preventing the model over fitting in specific places. Two UWB nodes were employed for the purpose of measurements, where one node acts as an anchor, and another node acts as a tag. The traces of NLOS and LOS channel measurements were solely evaluated without taking the reference positioning into consideration.

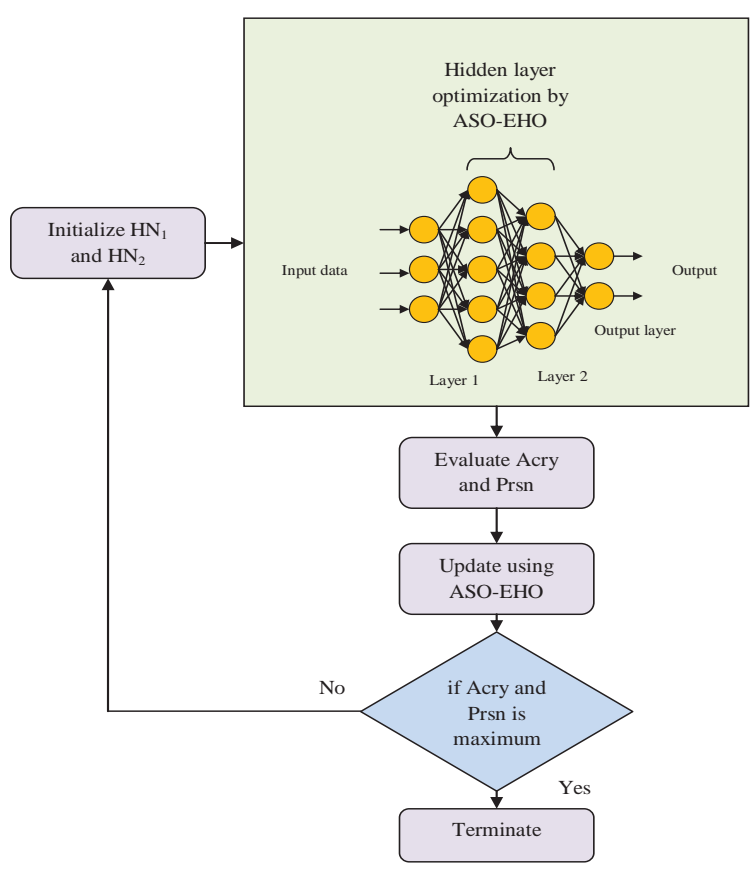

Fig. 3. Architecture of H-DNN for NLOS detection and range estimation

\section{Heuristic-based Deep Neural Network for NLOS Detection and Range Estimation}

The traditional DNN suffers from shortcomings such as it needs a huge quantity of data, highly expensive for the process of training the complex data models, it does not have the capability of providing the arguments in reaching a specific conclusion. In order to overcome these drawbacks, H-DNN is proposed, in which an improvement is made by optimizing its hidden neurons, thereby maximizing the accuracy as well as precision. The H-DNN is used for classifying the NLOS channel. It is also used to estimate the range of each node. DNN [22] is composed of three layers such as, input layer, output layer, and hidden layers. The DNN is shaped using two hidden layers for learning the mapping association among the input as well as the output data. The enhancement in the training iterations fits the decision boundary of the labelled training data in a continuous manner. The DNN's training speed as well as the classification accuracy's training speed is improved by constructing two hidden layers. In the hidden layer, the total count of nodes is estimated as

$$
n g=\sqrt{a g+b g}+c g
$$

where, $a g$ is the count of input layer nodes, $n g$ is the count of hidden layer nodes, $b g$ is the count of output layer nodes, and $c g$ is a constant with value between 0 to 10 . To enable the nonlinear fitness capabilities, an activation function is integrated into the DNN's hidden layer. The sigmoid is employed as an activation function and is given by

$$
S G=\frac{1}{1+e^{-x g}}
$$

The input data is given as $x g$, and it is activated using the mapping function $M G_{f g}$.

$$
M G_{f g}=\operatorname{signm}\left(\omega_{i g} x g+\beta_{i g}\right)
$$

Here, the weight matrix is represented by $\omega$, and the bias among the output and the hidden layer is represented by $\beta$. The suggested ASO-EHO is used to optimize the hidden neurons of DNN with the goal of enhancing accuracy and precision. The objective function related to the H-DNN for the NLOS detection and range estimation is given by

$$
O b j=\underset{\left\{H N_{1}, H N_{2}\right\}}{\arg \max }(\text { Acry }+ \text { Prsn })
$$

where, the accuracy is denoted by Acry, the precision is denoted by Prsn, and the hidden neurons of two layers used in DNN are denoted by $H N_{1}$ and $H N_{2}$ respectively. The bounding limit of the hidden neurons lies in between (555). Accuracy is defined as the discrepancy in the recognized outcome to the ground value. It is mathematically modeled as

$$
A c r y=\frac{P O^{T}+N E^{T}}{P O^{T}+N E^{T}+P O^{F}+N E^{F}}
$$

where, the terms $P O^{T}, N E^{T}, P O^{F}$, and $N E^{F}$ represent the true positive, true negative, false positive, and false negative respectively. The precision is defined as the measure of the 
deviation in the data retrieval from the original data. It is modeled as

$$
\text { Prsan }=\frac{P O^{T}}{P O^{T}+P O^{F}}
$$

The architecture of the H-DNN based NLOS detection and range estimation is depicted in Fig. 3.

\section{NODE LOCALIZATION USING ASO-EHO}

\section{A. Solution Encoding}

Fig. 4 depicts the solution encoding. The solution consists of a percentage of the distance in meters that the node's current position is to be relocated. The term $W_{n}$ defines the percentage

\begin{tabular}{|l|l|l|l|l|}
\hline $\mathrm{W}_{1}$ & $\mathrm{~W}_{2}$ & $\mathrm{~W}_{3}$ & $\cdots$ & $\mathrm{W}_{\mathrm{NG}}$ \\
\hline
\end{tabular}

Fig. 4. Solution encoding of node location

of distance in meter and its bounding limit ranges between ($20 \%$ to $20 \%$ ), where $n=1,2, \ldots, N G$ and $N G$ denotes the maximum count of nodes.

\section{B. Multi-objective Function}

In the developed anchor-free localization under NLOS conditions, the multi-objective function focuses on constraints such as energy overhead, hardware cost, and localization error. The minimization fitness function of the developed localization technique is given by

$$
\text { Obe }_{\text {new }}=\operatorname{Min}\left(\operatorname{Err}+E_{T}+\text { Hardware cost }\right)
$$

\section{Proposed ASO-EHO}

The proposed ASO-SHO is used to optimize the hidden neurons of the H-DNN with the intention of maximizing accuracy and precision. This proposed adaptive algorithm also helps for multi-objective anchor-free node localization. EHO [23] is a population-oriented algorithm that accomplishes the task on the basis of the herding characteristics of the elephants. The herding characteristics of the elephants is employed by two operators like separating operator and clan updating operator that are categorized for providing a format of global optimization approach. The elephant population is composed of few clans or groups and every clan is composed of the identical elephant count. Elephants jointly reside by obeying the matriarch's instructions, referred to as the clan updating operator. A selected male elephant count stop their clan during the ageing process called separating operator. The elephants present in the clan are treated as search particle in a population size of $N E$ having de dimension habitat. The site of every element $i e$ at iteration te is described by $E E_{i e}^{t e}$, and the elephant population of size $N E$ at iteration te is given by

$$
E E_{i e}^{t e}=\left[E E_{i e, 1}^{t e}, E E_{i e, 2}^{t e}, \cdots, E E_{i e, d e}^{t e}\right]
$$

where $i e=1,2,3, \ldots, N E$ and $t e=1,2, \ldots t e_{\max }$ (iteration).

$$
E E=\left[\begin{array}{ccccc}
E E_{11}^{t e} & E E_{12}^{t e} & E E_{13}^{t e} & \cdots & E E_{1 d e}^{t e} \\
E E_{21}^{t e} & E E_{22}^{t e} & E E_{23}^{t e} & \cdots & E E_{2 d e}^{t e} \\
E E_{21}^{t e} & E E_{32}^{t e} & E E_{33}^{t e} & \cdots & E E_{3 d e}^{t e} \\
\cdot & \cdot & \cdot & \cdots & \cdot \\
\cdot & \cdot & \cdot & \cdots & \cdot \\
\cdot & \cdot & \cdot & \cdots & \cdot \\
E E_{N E 1}^{t e} & E E_{N E 2}^{t e} & E E_{N E 3}^{t e} & \cdots & E E_{N E d e}^{t e}
\end{array}\right]_{(14)}
$$

Since elephants jointly reside by the matriarch instructions called best $E E_{i e}$, every elephant of clan $E E_{i e}$ update its subsequent position that is effected by the matriarch position.

$$
E E_{i e}^{t e+1}=E E_{i e}^{t e}+\alpha \times\left(E E_{b e s t}^{t e}-E E_{i e}^{t e}\right) \times r e
$$

where, term $r e \in[0,1]$ represents a random number, the best elephant present in the clan is represented by $E E_{\text {best }}$, a scale factor describing the matriarch effect is represented by $\alpha \in[0,1]$, the older position of elephant $i e$ is represented by $E E_{i e}^{t e}$, and the recently varied position of elephant $i e$ is represented by $E E_{i e}^{t e+1}$. The matriarch elephant in every clan was not possible to vary using (15). In the case of best one, it is updated as

$$
E E_{\text {best }}^{t e+1}=\beta \times E E_{\text {center }}^{\text {te }}
$$

Here, the term $\beta \in[0,1]$ defines a factor that denotes the influence factor of $E E_{\text {best }}$, and $E E_{\text {center }}$ represents the centre of clan $E E_{i e}$ and it is based on ne_clan that denotes the elephant count in clan $E E_{i e}$ as given by

$$
E E_{\text {center }}^{\text {te }}=\frac{1}{n e_{-} \text {clan }} \times \sum_{i e=1}^{n e_{-} \text {clan }} E E_{i e}^{t e}
$$

When male elephants stop their clan and isolate live during the ageing process, the process of separating can be modelled using the separating operator. Moreover, the enhancement in the exploring capability of EHO utilizes the worst fitness elephant individual as the separating operator in each iteration and is defined as

$$
E E_{\text {worst }}^{t e}=L E+(U E-L E+1) \times r e
$$

Here, the lower and upper bounds of the site of elephant individuals are represented by $L E$ and $U E$, the random number is represented by $r e$, and the worst elephant individual present in the clan $E E_{i e}$ is represented by $E E_{\text {worst }}$.

Generally, in the traditional EHO, if $\left(E E_{i e} \neq E E_{\text {worst }}\right)$, then the clan updating operator is updated using (15). Otherwise, the separating operator is updated using (18). However, in the proposed ASO-EHO, if $\left(E E_{i e} \neq E E_{\text {worst }}\right)$, then update takes place using the same clan updating operator as in (15). Otherwise, the update takes place using the adaptive separating operator equation given by

$$
E E_{\text {new }}^{t e}=E E_{i e}+\lambda\left(E E_{\text {worst }}^{t e}-E E_{i e}\right) \times r e
$$

Here, the random value re is defined between 0 and 1 , and the term $\lambda$ is computed as

$$
\lambda=\frac{\text { Worst } f i t}{\text { mean }(\text { fit })}
$$


In the above equation, the fitness is represented by the term fit. The pseudo code of the ASO-EHO is shown in Algorithm 1 and the flowchart of the ASO-SHO is given in Fig. 5.

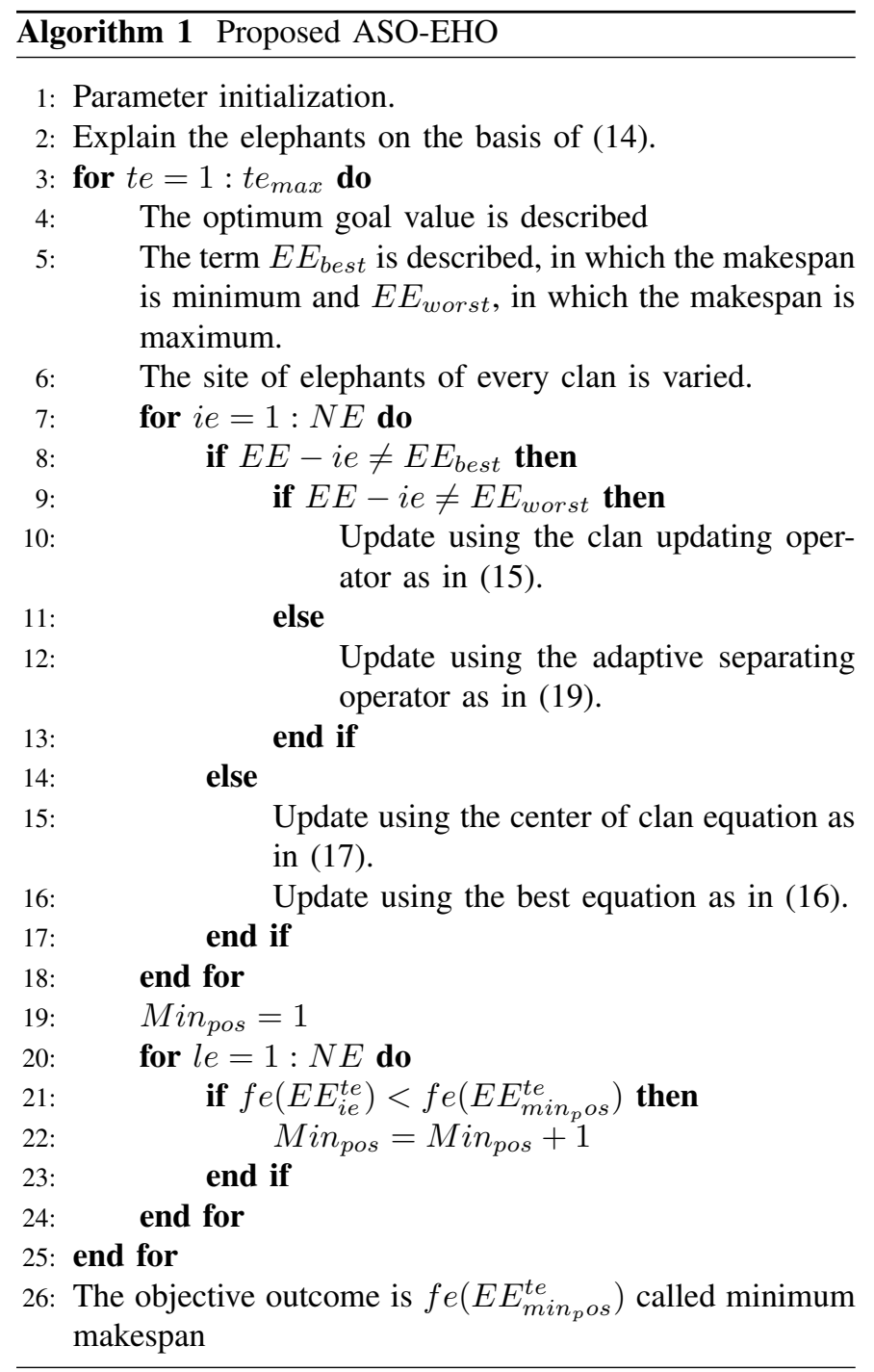

\section{RESUlts AND Discussions}

\section{A. Experimental Setup}

The proposed anchor-free node localization in WSN under NLOS environment was implemented in MATLAB R2019a and experimental analysis was carried out. The dataset was gathered from the standard publically available UWB LOS and NLOS dataset. The proposed model's performance is evaluated by comparing it to traditional methods in terms of several metrics such as localization error, hardware cost, and energy overhead. The population size was considered as 10 and the maximum performed iterations were 1000 . The simulation parameters are given in Table I. The developed ASO-EHOH-DNN is compared with the optimization algorithms such as MFO-H-DNN [24], SHO-H-DNN [25], JA-H-DNN [26], and EHO-H-DNN [23] and machine learning models like CNN [27]], Auto Encoder [28], RF [29], SVM [30], KNN [31], and NN [32].

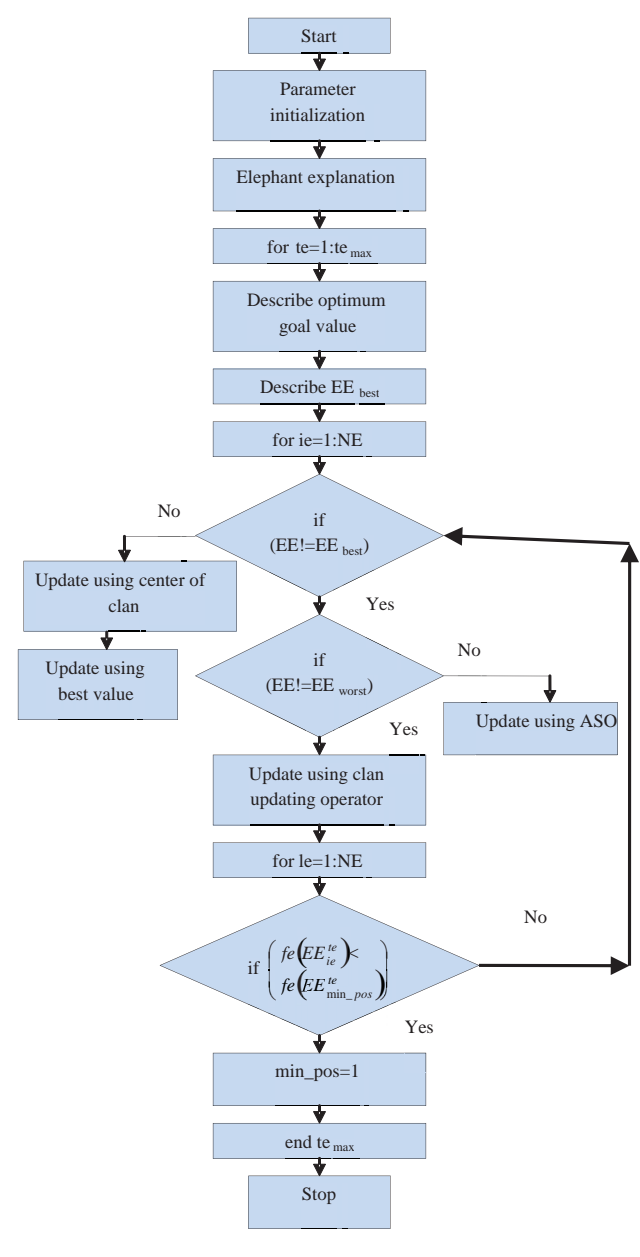

Fig. 5. : Flow Chart for the proposed ASO-EHO

TABLE I

SiMUlation PARAMETERS

\begin{tabular}{ll}
\hline Parameter & Value \\
\hline Number of sensors & $50-350$ \\
Network grid & From $(0,0)$ to $(600,600) \mathrm{m}$ \\
Sink-1 & At $(700,200) \mathrm{m}$ \\
Sink-2 & At $(700,400) \mathrm{m}$ \\
Initial energy & $1 \mathrm{~J}$ \\
$E_{\text {elec }}$ & $50 \mathrm{~nJ} / \mathrm{bit}$ \\
$\epsilon_{f s}$ & $10 \mathrm{pJ} / \mathrm{bit} / \mathrm{m}^{2}$ \\
$\epsilon_{\text {amp }}$ & $0.0013 \mathrm{pJ} / \mathrm{bit} / \mathrm{m}^{4}$ \\
$d_{0}$ & $87 \mathrm{~m}$ \\
\hline
\end{tabular}

\section{B. Performance Metrics}

The performance measures used here are described below. (a) Specificity: It expresses the rate of the wrong data correctly neglected during the data retrieval.

$$
\text { Speciy }=\frac{N E^{T}}{P O^{F}+N E^{T}}
$$

(b) FPR: It refers to the total positive results within the negative output.

$$
F P R=\frac{P O^{F}}{P O^{F}+N E^{T}}
$$




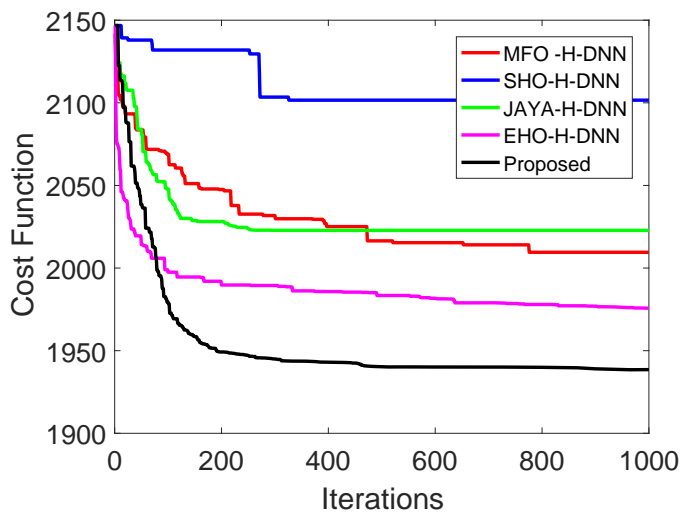

(a)

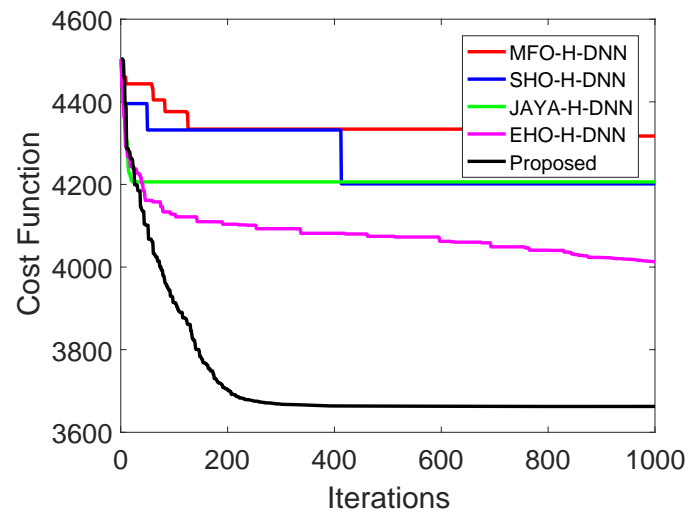

(b)

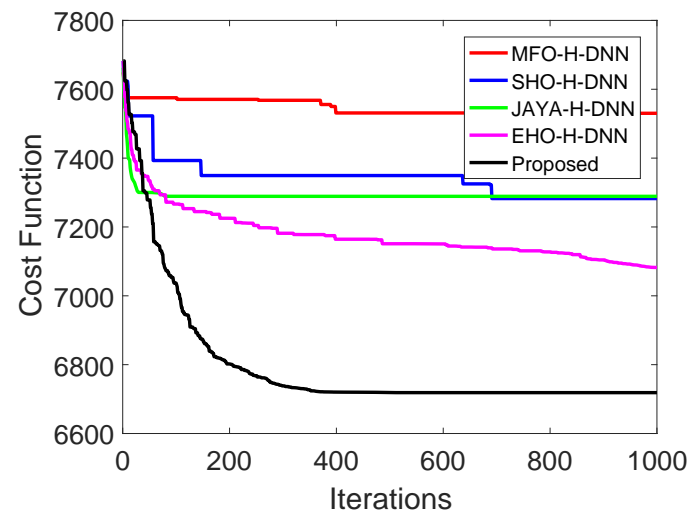

(c)

Fig. 6. Convergence analysis of the developed and traditional anchor-free node localization in WSN with (a) 100 nodes, (b) 200 nodes, (c) 300 nodes

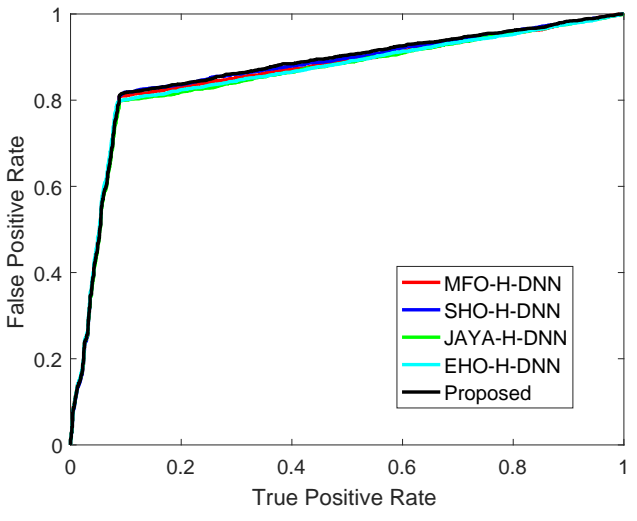

(a)

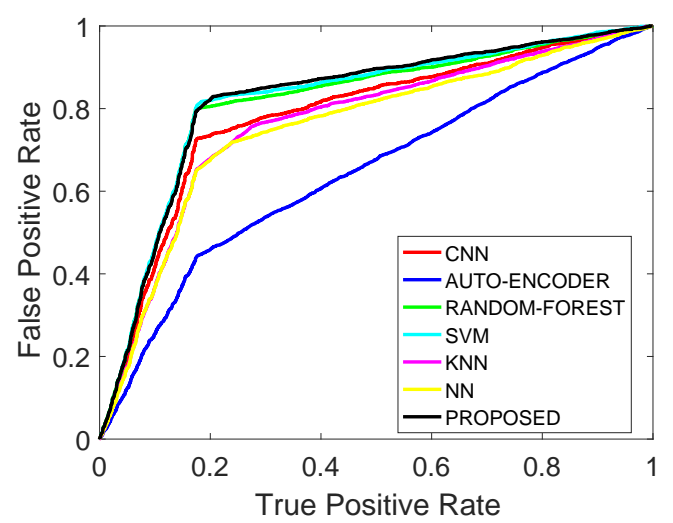

(b)

Fig. 7. (a) ROC Analysis in terms of NLOS detection. (b) ROC Analysis in terms of range estimation

(c) Accuracy: It is described in (10).

(d) MCC: It is the correlation coefficient between the response parameters.

$$
M C C=\frac{P O^{T} \times N E^{T}-P O^{F} \times N E^{F}}{\sqrt{\left(P O^{T}+P O^{F}\right)\left(P O^{T}+N E^{F}\right)}}
$$

(e) FDR: It refers to the total false positives within the positive values

$$
F D R=\frac{P O^{F}}{P O^{F}+P O^{T}} .
$$

(f) Precision: It is described in (11).

(g) F1 Score:It is the mean value between sensitivity and specificity.

$$
F 1 s c o r e=\frac{\text { Sensy } \times \text { Prsn }}{\text { Sensy }+ \text { Prsn }}
$$


TABLE II

PERFORMANCE ANALYSIS IN TERMS OF NLOS DETECTION FOR HEURISTIC ALGORITHMS

\begin{tabular}{ccccccccccc}
\hline & NPV & Specificity & FPR & Accuracy & F1 Score & Precision & MCC & FDR & Sensitivity & FNR \\
\hline MFO-H-DNN & 0.91155 & 0.891155 & 0.088454 & 0.86167 & 0.85114 & 0.89735 & 0.72593 & 0.10265 & 0.80945 & 0.19055 \\
SHO-H-DNN & 0.91061 & 0.91061 & 0.089385 & 0.86524 & 0.85569 & 0.89733 & 0.73257 & 0.10267 & 0.81774 & 0.18226 \\
JA-H-DNN & 0.91341 & 0.91341 & 0.08659 & 0.86524 & 0.85524 & 0.89989 & 0.73292 & 0.10011 & 0.81481 & 0.18519 \\
EHO-H-DNN & 0.9027 & 0.9027 & 0.0973 & 0.85143 & 0.83992 & 0.88678 & 0.70545 & 0.11322 & 0.79776 & 0.20224 \\
ASO-EHO-H-DNN & 0.93529 & 0.93529 & 0.064711 & 0.86619 & 0.85288 & 0.92138 & 0.73824 & 0.07862 & 0.79386 & 0.20614 \\
\hline
\end{tabular}

TABLE III

PERFORMANCE ANALYSIS IN TERMS OF NLOS DETECTION FOR MACHINE LEARNING AND DEEP LEARNING ALGORITHMS

\begin{tabular}{ccccccccccc}
\hline & F1 Score & Precision & FDR & Sensitivity & FNR & MCC & FPR & Accuracy & NPV & Specificity \\
\hline CNN & 0.76103 & 0.79829 & 0.20171 & 0.7271 & 0.2729 & 0.55481 & 0.17551 & 0.7769 & 0.82449 & 0.82449 \\
Auto Encoder & 0.001945 & 0.4 & 0.6 & 0.000975 & 0.99903 & 0.00612 & 0.001397 & 0.51119 & 0.9986 & 0.9986 \\
RF & 0.84901 & 0.87008 & 0.12992 & 0.82895 & 0.17105 & 0.71221 & 0.11825 & 0.85595 & 0.88175 & 0.88175 \\
SVM & 0.84982 & 0.88624 & 0.11376 & 0.81628 & 0.18372 & 0.7196 & 0.10009 & 0.85905 & 0.89991 & 0.89991 \\
KNN & 0.24511 & 0.24607 & 0.75393 & 0.24415 & 0.75585 & 0.47056 & 0.71462 & 0.26524 & 0.28538 & 0.28538 \\
NN & 0.71842 & 0.71755 & 0.28245 & 0.7193 & 0.2807 & 0.44879 & 0.27048 & 0.72452 & 0.72952 & 0.72952 \\
ASO-EHO-H-DNN & 0.85524 & 0.89989 & 0.10011 & 0.1481 & 0.18519 & 0.73292 & 0.086594 & 0.86524 & 0.91341 & 0.91341 \\
\hline
\end{tabular}

TABLE IV

PERFORMANCE ANALYSIS IN TERMS OF RANGE ESTIMATION FOR HEURISTIC ALGORITHMS

\begin{tabular}{ccccccccc}
\hline & L1 Norm & MASE & L-Infinity Norm & SMAPE & RMSE & L2 Norm & MAE & MEP \\
\hline MFO-H-DNN & 4518.9 & 0.52599 & 6.6406 & 0.33678 & 1.3999 & 90.721 & 1.0759 & 53.456 \\
SHO-H-DNN & 4417.1 & 0.52274 & 6.5354 & 0.33148 & 1.3819 & 89.557 & 1.0517 & 51.588 \\
JA-H-DNN & 5162.1 & 0.64254 & 8.1747 & 0.373374 & 1.5757 & 102.12 & 1.2291 & 57.059 \\
EHO-H-DNN & 4377.4 & 0.49146 & 6.6055 & 0.32446 & 1.373 & 88.984 & 1.0422 & 48.642 \\
ASO-EHO-H-DNN & 4220.6 & 0.46225 & 6.8954 & 0.31607 & 1.3378 & 86.701 & 1.0049 & 45.783 \\
\hline
\end{tabular}

TABLE V

PERFORMANCE ANALYSIS IN TERMS OF RANGE ESTIMATION FOR MACHINE LEARNING AND DEEP LEARNING ALGORITHMS

\begin{tabular}{ccccccccc}
\hline & RMSE & SMAPE & L-Infinity Norm & MASE & L2 Norm & MAE & L1 Norm & MEP \\
\hline SVM & 0.44937 & 0.23814 & 0.49998 & 0.17097 & 29.122 & 0.44756 & 1879.7 & 49.969 \\
Auto Encoder & 0.54782 & 0.29565 & 0.6 & 0.20834 & 35.503 & 0.5454 & 2290.7 & 110.42 \\
RF & 0.62044 & 0.33818 & 0.65 & 0.23607 & 40.209 & 0.61798 & 2595.5 & 333.5 \\
CNN [37] & 1.5144 & 0.36819 & 6.9461 & 0.44188 & 98.143 & 1.1567 & 4858.3 & 46.61 \\
KNN & 3.5111 & 0.77259 & 14.354 & 1.0553 & 227.54 & 2.7625 & 1160.2 & 589.73 \\
NN & 2.5402 & 0.54843 & 9.78 & 0.73302 & 164.62 & 1.9189 & 8059.3 & 76.52 \\
ASO-EHO-H-DNN & 1.3378 & 0.31607 & 6.8954 & 0.46225 & 86.701 & 1.0049 & 4220.6 & 45.783 \\
\hline
\end{tabular}

(h) Sensitivity: It refers to the correctly identified retrieved data among the various data.

$$
\text { Sensy } / T P R=\frac{P O^{T}}{P O^{T}+N E^{F}}
$$

(i) NPV: It indicates the direct false values from the retrieval system.

$$
N P V=\frac{N E^{T}}{N E^{F}+N E^{T}}
$$

(j) FNR: It refers to the rate of negative response within the positive values.

$$
F N R=\frac{N E^{F}}{N E^{F}+P O^{T}}
$$

The various error measures used here are described below.

(a) MAE: It is a measure of difference between two continuous variables.

$$
M A E=\frac{\sum_{e i q=1}^{n j q} \mid f_{o r} r_{e i q}-\text { for }_{e i q} \mid}{n j q}
$$

(b) L Infinity Norm: The length of a vector can be calculated using the maximum norm.

$$
L Q_{\text {inf }}=\max _{1 \leq e i q \leq n j q}\left|L Q_{e i q}\right|
$$

(c) RMSE: It is a frequently used measure of the differences between values predicted by a model or an estimator and the values observed.

$$
R M S E=\sqrt{\frac{\sum_{e i q=1}^{n j q}\left(f o q_{e i q 2}-f o q_{e i q 1}\right)^{2}}{n j q}}
$$

(d) SMAPE: It is an accuracy measure based on percentage errors.

$$
S M A P E=\frac{100 \%}{n j q} \sum_{e i q=1}^{n j q} \frac{|f o q-a c q|}{\frac{(|a c q|+|f o q|)}{2}}
$$

Here, the value of computation added for each fitted point is described by eiq, the count of fitted points is described as $n j q$, the actual value is given as $a c q$, and the predicted value is given as foq.

(e) L1 Norm: It is the sum of the magnitudes of the vectors in a space.

$$
L Q_{1}=\sum_{e i q}\left|L Q_{e i q}\right|
$$

Here, $L Q$ is a matrix, eiq $=1,2, \ldots, E i q$, and Eiq is the size of the matrix. 


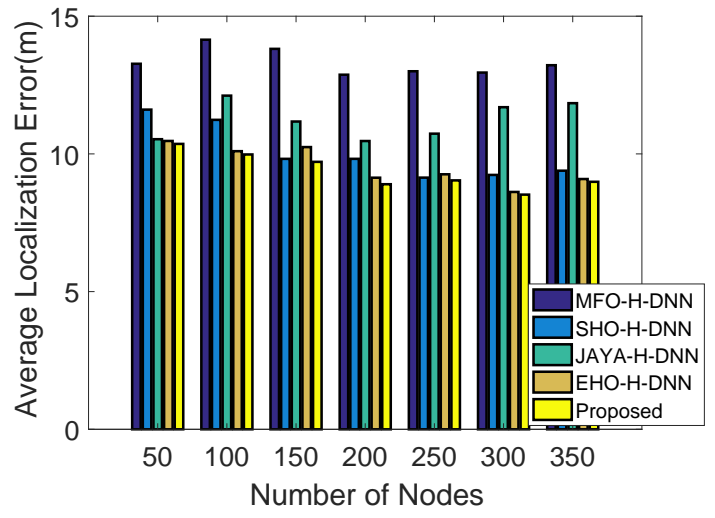

(a)

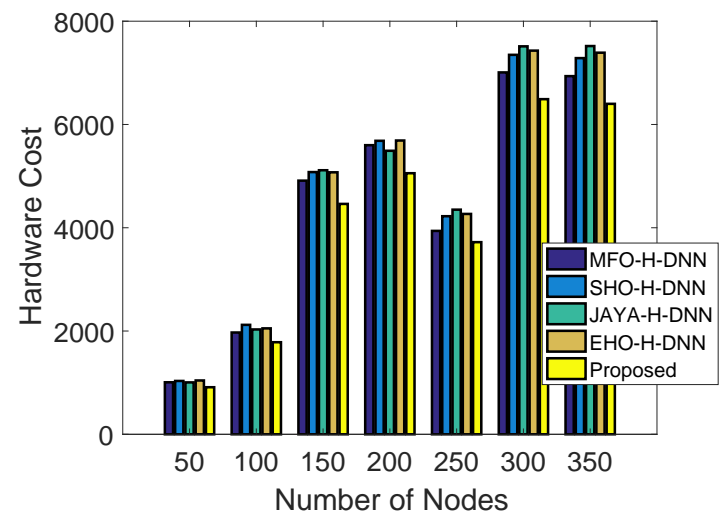

(b)

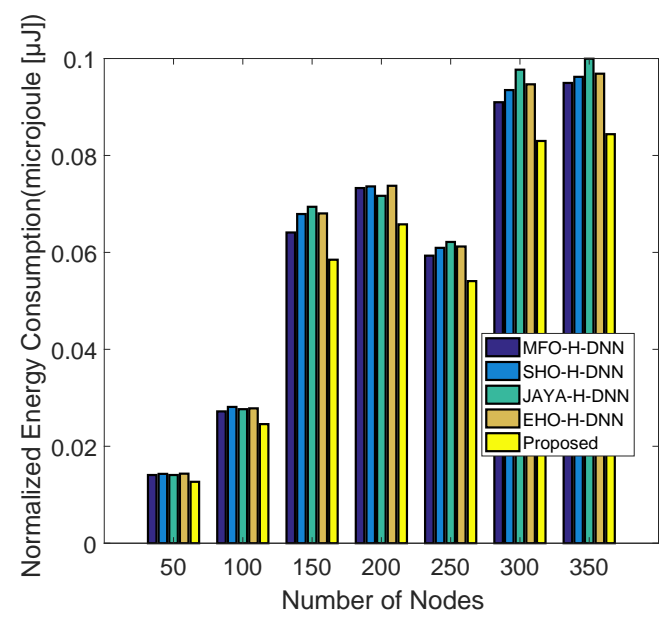

(c)

Fig. 8. Performance analysis

(f) MASE: It is the mean absolute error of the forecast values, divided by the mean absolute error of the in-sample one-step naive forecast.

$$
M A S E=\operatorname{mean}\left(\frac{|f o q|}{\frac{1}{n j q-1} \sum_{e i q=1}^{n j q}\left|a c q_{e i q}-a c q_{e i q-1}\right|}\right)
$$

(g) L2 Norm: It is the shortest distance to go from one point to another. It is also taken as Euclidean norm.

$$
L Q 2=\left(\sum_{e i q=1}^{n j q} L Q_{e i q}^{2}\right)^{\frac{1}{2}}
$$

(h) MEP: the MEP is the computed average of percentage errors by which forecasts of a model differ from actual values of the quantity being forecast.

$$
M E P=\frac{100 \%}{n j q} \sum_{e i q=1}^{n j q} \frac{a c q-f o q}{a c q}
$$

\section{Convergence Analysis}

Fig. 6 is the comparison of cost function about the convergence rate between MFO-H-DNN, SFO-H-DNN, JA-HDNN, EHO-H-DNN, and the proposed scheme. The nodes are varied from 100 to 300 to show the scalability of the proposed scheme. The EHO algorithm has a weakness in terms of its lack of exploitation, which causes it to converge slowly. The exploration/exploitation abilities of the proposed ASO-EHOH-DNN algorithms are achieved by updating the two operators (clan and separation operator). In our suggested ASO-EHOH-DNN, we improve the separation operator and offer a new separation strategy that can build new local optimums in search space, improve the algorithm's search ability, and produce an algorithm with the best exploration-exploitation balance.The results clearly indicate that ASO-EHO-H-DNN is capable of accelerating the convergence rate effectively and can develop better solutions compared to the MFO-H- DNN, SHO-H-DNN, JA-H-DNN, and EHO-H-DNN. Our proposed ASO-EHO-H-DNN algorithm maintains an efficient balance between exploitation and exploration phases and converges faster. The suggested ASO-EHO-H-DNN also achieves a lower cost function value than any other classic optimization methods.

\section{ROC Analysis}

When analysing the performance of binary classification methods, the receiver operating characteristic (ROC) curve is typically utilised. Rather than a single value like most other 
metrics, it displays a graphical representation of a classifier's performance. The ROC analysis on NLOS detection is shown in Fig. 7(a) in terms of TPR and FPR. The proposed ASOEHO-H-DNN outperforms in terms of TPR and FPR. For instance, at TPR $=0.4$, the FPR of the ASO-EHO-H-DNN is $4.76 \%, 3.53 \%, 7.32 \%$, and $6.02 \%$ better than MFO-HDNN, SFO-H-DNN, JA-H-DNN, and EHO-H-DNN respectively. Therefore, the NLOS detection is performed better with the suggested ASO-EHO-H-DNN than all the traditional methods.

Fig.7(b) shows the ROC analysis for range estimation. The proposed ASO-EHO-H-DNN produces better results. For instance, at TPR $=0.6$, the FPR of the ASO-EHO-H-DNN is $7.14 \%, 28.57 \%, 2.27 \%, 1.12 \%, 9.76 \%$, and $12.5 \%$ higher than $\mathrm{CNN}$, Auto Encoder, RF, SVM, KNN, and NN respectively. Thus, the developed ASO-EHO-H-DNN outperforms state-ofthe-art approaches in terms of range estimation.

\section{E. Performance Analysis}

The performance analysis of NLOS detection is displayed in Table 2 and 3, and ASO-EHO-H-DNN is found to be better than any other standard approach. The performance analysis with respect to the range estimation is depicted in Table 4 . The classifier analysis with respect to the range estimation is given in Table 5. In terms of range estimation, it is clear that the proposed ASO-EHO-H-DNN outperforms all other established approaches.

By altering the number of nodes from 50 to 350, the proposed anchor-free node localization is evaluated with a multiobjective function utilising different optimization algorithms. In Fig. 8(a), for instance, at 350 nodes, the locatlization error of ASO-EHO-H-DNN is $38.46 \%, 15.79 \%, 29.17 \%$, and $5.88 \%$ lower than MFO-H-DNN, SFO-H-DNN, JA-HDNN, and EHO-H-DNN respectively. The reason is that the proposed H-DNN performs better LOS/NLOS classification and range estimation. Furthermore, 6shows that ASO-EHO$\mathrm{H}-\mathrm{DNN}$ performs better in terms of node localization. It is also observed that the performance of EHO-H-DNN is close to that of the proposed approach; however, the hardware cost and energy overhead of EHO-H-DNN are much greater than those of the proposed method.

As dipicted in Fig. 8(b), at 300 nodes, the hardware cost of ASO-EFO-H-DNN is $13.96 \%, 14.20 \%, 14.45 \%$, and $14.33 \%$ less than MFO-H-DNN, SFO-H-DNN, JA-H-DNN, and EHOH-DNN respectively. In Fig. 8(c), the energy overhead for the ASO-EHO-H-DNN at 350 nodes is $10.64 \%, 11.58 \%, 16 \%$, and $12.5 \%$ less than MFO-H-DNN, SFO-H-DNN, JA-H-DNN, and EHO-H-DNN respectively. As a conclusion, the developed ASO-EHO-H-DNN algorithm improves the localization model's overall performance.

\section{CONCLUSION}

In this paper, an anchor-free node localization model for multi-sink WSN under NLOS conditions is developed. The experiment relied on data from the standard UWB LOS and NLOS datasets. For the dimensionality reduction, PCA was used. The first phase used a new H-DNN for NLOS/LOS channel classification. The H-DNN was also used to estimate the range for the LOS node. The ASO-EHO was used to optimise the hidden neurons of DNN in order to improve accuracy and precision. The anchor-free node localization was completed in the final step by taking into account a multiobjective function with objectives such as localization error, hardware cost, and energy overhead. The simulation results show that the proposed ASO-EHO-H-DNN outperforms all other state-of-the-art methods for NLOS detection and range estimation for anchor-free node localization in WSN.

\section{REFERENCES}

[1] Shu F, Yang S P, Qin Y L, et al. "Approximate analytic quadraticoptimization solution for TDOA-based passive multi-satellite localization with earth constraint”, IEEE Access, vol. 4, pp. 9283-9292, 2016.

[2] J. Yin, Q. Wan, S. Yang, and K. C. Ho. A simple and accurate tdoa-aoa localization method using two stations. IEEE Signal Processing Letters, 23(1):144-148, 2016.

[3] N. H. Nguyen and K. Doganay. Optimal geometry analysis for multistatic toa localization. IEEE Transactions on Signal Processing, 64(16):41804193, 2016.

[4] G. Wang and K. Yang. A new approach to sensor node localization using rss measure-ents in wireless sensor networks. IEEE Transactions on Wireless Communications,10(5):1389-1395, 2011.

[5] C. Huang et al., Machine Learning-Enabled LOS/NLOS Identification for MIMO Systems in Dynamic Environments, in IEEE Transactions on Wireless Communications, vol. 19, no. 6, pp. 3643-3657, June 2020.

[6] H.-S. Ahn and W. Yu, Environmental-adaptive RSSI-based indoor localization, IEEE Trans. Autom. Sci. Eng., vol. 6, no. 4, pp. 626-633, Oct. 2009.

[7] K. Wu, J. Xiao, Y. Yi, D. Chen, X. Luo, and L. M. Ni, CSI-based indoor localization, IEEE Trans. Parallel Distrib. Syst., vol. 24, no. 7, pp. 13001309, Jul. 2013.

[8] Chan Y T, Tsui W Y, So H C, et al. Time-of-arrival based localization under NLOS conditions, IEEE Trans Veh Technol, vol. 55, pp. 17-24, 2006.

[9] Wang J., Zhang X., Gao Q., Yue H., Wang H. Device-free wireless localization and activity recognition: A deep learning approach. IEEE Trans. Veh. Technol. vol. 66, pp. 62586267, 2017.

[10] Eman Saad, Mostafa Elhosseini, Amira Yassin Haikal, Recent achievements in sensor localization algorithms, Alexandria Engineering Journal, Volume 57, Issue 4,pp. 4219-4228, 2018.

[11] Klemen Bregar AND Mihal Mohorfif, Improving Indoor Localization Using Convolutional Neural Networks on Computationally Restricted Devices, IEEE Access, vol. 6, April 2018.

[12] Moses A. Koledoye, Tullio Facchinetti and Luis Almeida, "Improved MDS-based Localization with Non-line-of-sight RF Links", Journal of Intelligent and Robotic Systems, vol. 98, pp. 227-237, April 2019.

[13] Jingyu Hua, Yejia Yin, Anding Wang, Yu Zhang and Weidang Lu, Geometry-based non-line-of-sight error mitigation and localization in wireless communications", Science China Information Sciences, vol. 62, August 2019.

[14] Om Jee Pandey, Richika Sharan and Rajesh M. Hegde, Localization in Wireless Sensor Networks Using Visible Light in Non-Line of Sight Conditions, Wireless Personal Communications, vol. 97, pp. 6519-6539, August 2017.

[15] Xiaofeng Yang, Feng Zhao, Tiejun Chen, NLOS identification for UWB localization based on import vector machine, AEU - International Journal of Electronics and Communications, vol. 87, pp. 128-133, April 2018.

[16] Ardiansyah Musa, Gde Dharma Nugraha Hyojeong Han Deokjai Choi Seongho Seo Juseok Kim, A decision tree?based NLOS detection method for the UWB indoor location tracking accuracy improvement, International Journal of Communication Systems, April 2019.

[17] Yan Wang, Xuehan Wu, and Long Cheng, A Novel Non-Line-ofSight Indoor Localization Method for Wireless Sensor Networks, Hindawi Journal of Sensors, 2018.

[18] Wei Wang, Gang Wang, Member, IEEE, Jie Zhang, and Youming Li, Robust Weighted Least Squares Method for TOA-Based Localization Under Mixed LOS/NLOS Conditions, IEEE Communications Letters, vol. 21 , no. 10 , pp. 2226-2229, October 2017 
[19] Strumberger I., Beko M., Tuba M., Minovic M., Bacanin N. Elephant Herding Optimization Algorithm for Wireless Sensor Network Localization Problem. IFIP Advances in Information and Communication Technology, Springer, vol 521, pp. 174-184, 2018.

[20] W. Heinzelman, A. Chandrakasan, H. Balakrishnan, An applicationspecific protocol architecture for wireless microsensor networks, IEEE Transactions on Wireless Communications, 1 ,pp. 660 670, 2002.

[21] Klemen Bregar, Andrej Hrovat, and Mihael Mohorcic. Nlos channel detection with multilayer perceptron in low-rate personal area networks for indoor localization accuracy improvement. In Proceedings of the 8th Joef Stefan International Postgraduate School Students Conference, Ljubljana, Slovenia, 052016.

[22] S. Ramesh, D. Vydeki, Recognition and classification of paddy leaf diseases using Optimized Deep Neural network with Jaya algorithm, information processing in agriculture, 2019.

[23] Ronali Madhusmita, and SahooSasmita Kumari Padhy, "Elephant Herding Optimization for Multiprocessor Task Scheduling in Heterogeneous Environment", Computational Intelligence in Pattern Recognition, pp 217-229, February 2020.

[24] Seyedali Mirjalili, "Moth-flame optimization algorithm: A novel natureinspired heuristic paradigm", Knowledge-Based Systems, vol. 89, pp. 228-249, November 2015.

[25] Gaurav Dhiman, Vijay Kumar, "Spotted hyena optimizer: A novel bioinspired based metaheuristic technique for engineering applications", Advances in Engineering Software, vol. 114, pp. 48-70, December 2017.

[26] R. Venkata Rao, "Jaya: A simple and new optimization algorithm for solving constrained and unconstrained optimization problems", International Journal of Industrial Engineering Computations, vol. 7, pp. 19-34, 2016.

[27] Fernando Gama;Antonio G. Marques;Geert Leus;Alejandro Ribeiro, "Convolutional Neural Network Architectures for Signals Supported on Graphs", IEEE Transactions on Signal Processing, vol. 67, no. , 2019.

[28] Borui Hou;Jianyong Yang;Pu Wang;Ruqiang Yan, "LSTM-Based AutoEncoder Model for ECG Arrhythmias Classification", IEEE Transactions on Instrumentation and Measurement, vol. 69, no. 4, 2020.

[29] Mira Jeong;Jaeyeal Nam;Byoung Chul Ko, "Lightweight Multilayer Random Forests for Monitoring Driver Emotional Status", IEEE Access, vol. 8, 2020.

[30] Bohyung Han;Larry S. Davis, "Density-Based Multifeature Background Subtraction with Support Vector Machine", IEEE Transactions on Pattern Analysis and Machine Intelligence, vol. 34, no. 5, 2012.

[31] J. P. Jose, P. Poornima and K. M. Kumar, "A novel method for color face recognition using KNN classifier," 2012 International Conference on Computing, Communication and Applications, Dindigul, Tamilnadu, pp. $1-3,2012$.

[32] Shang-Hung Lin, Sun-Yuan Kung and Long-Ji Lin, "Face recognition/detection by probabilistic decision-based neural network," in IEEE Transactions on Neural Networks, vol. 8, no. 1, pp. 114-132, Jan. 1997.

[33] P. Chatterjee and N. Das, Multiple sink deployment in multi-hop wireless sensor networks to enhance lifetime, 2015 Applications and Innovations in Mobile Computing (AIMoC), 2015, pp. 48-54,

[34] Xiuwen Fu, Yongsheng Yang,Modeling and analysis of cascading nodelink failures in multi-sink wireless sensor networks, Reliability Engineering \& System Safety, Volume 197, 2020, 